\title{
Clinical Decision-Making: Observing the Smartphone User, an Observational Study in Predicting Acute Surgical Patients' Suitability for Discharge
}

\author{
Richard Hoffmann, MBBS*, Simon Harley, MBBS, Samuel Ellison, MBBS, Peter G. Devitt, MBBS, FRACS
}

Department of Surgery, Royal Adelaide Hospital, Adelaide, South Australia, Australia.

\begin{abstract}
INTRODUCTION: An accurate and rapid assessment of an acutely unwell patient's clinical status is paramount for the physician. There is an increasing trend to rely on investigations and results to inform a clinician of a patient's clinical status, with the subtleties of clinical observation often ignored. The aim of this study was to determine if a patient's use of a smartphone during the initial clinical assessment by a surgical consultant could be used as a surrogate marker for patient well-being, represented as their suitability for same-day discharge.
\end{abstract}

METHODS: This was a prospective observational study performed over 2 periods at a tertiary hospital in South Australia. All patients admitted by junior surgical doctors from the emergency department to the acute surgical unit were eligible for inclusion. Upon consultant review, their status as a smartphone user was recorded in addition to their duration of hospital stay and basic demographic data. All patients and all but 1 of the consultants were blinded to the trial.

RESULTS: Two hundred and twenty-one patients were eligible for inclusion. Of these patients, $11.3 \%$ were observed to be using a smartphone and $23.5 \%$ of patients were discharged home on day 1 . Those who were observed to be using a smartphone were 5.29 times more likely to be discharged home on day 1 and were less likely to be subsequently readmitted.

CONCLUSIONS: The addition of the smartphone sign to a surgeon's clinical acumen can provide yet another tool in aiding the decision for suitability for discharge. Journal of Hospital Medicine 2018;13:21-25. Published online first August 23, 2017. (C) 2018 Society of Hospital Medicine
T he value placed on bedside clinical observation in the decision-making process of a patient's illness has been diminished by today's armamentarium of sophisticated technology. Increasing reliance is now placed on the result of nonspecific tests in preference to bedside clinical judgement in the diagnostic and management process. While diagnostic investigations have undoubtedly provided great advancements in medical care, they come at time and financial costs. Physicians should therefore continue to be encouraged to make clinical decisions based on their bedside assessment.

With hospital overcrowding a significant problem within the healthcare system and the expectation that it will worsen with an ageing population, identifying factors that predict patient suitability for discharge has become an important focus for clinicians. ${ }^{1,2}$ There exists a paucity of literature predicting discharge suitability of general surgical patients admitted through the emergency department (ED). Furthermore, despite the extensive research into the effectiveness of discharge planning, ${ }^{3}$ little research has been conducted to describe positive predic-

\footnotetext{
*Address for correspondence and reprint requests: Richard Hoffmann, MBBS, Department of Surgery, Level 5, Eleanor Harrald Building, Royal Adelaide Hospital, Adelaide, South Australia 5000; Telephone: +61-8-8222-5516; Fax: +61-8-8222-5896; E-mail: richard.hoffmann@sa.gov.au
}

Received: February 21, 2017; Revised: June 2, 2017;

Accepted: June 19, 2017

2018 Society of Hospital Medicine DOI: 10.12788/jhm.2797 tive indicators for discharge. Observations made during surgical rounds have led the authors to consider that individuals who are using a smartphone during their bedside assessment may be clinically well enough for discharge.

The aim of this study was to assess whether the clinical assessment of an acute surgical patient could be usefully augmented by the observation of the active use of smartphones (the smartphone sign) and whether this could be used as a surrogate marker to indicate a patient's well-being and suitability for sameday discharge from the hospital in acute surgical patients.

\section{METHODS}

\section{Design and Setting}

This was a prospective observational study performed over 2 periods at a tertiary hospital in South Australia, Australia. At our institution, acute surgical patients are admitted to the acute surgical unit (ASU) from the ED by junior surgical doctors. Patients are then reviewed by the on-call surgical consultant, who implements management plans or advises discharge on 2 occasions per day.

\section{Participants}

All patients admitted under the ASU were considered eligible for the study. Exclusion criteria included patients that (i) required immediate surgical intervention (defined as time of review to theatre of less than 4 hours) and (ii) had immediate admission to the intensive care unit. 
Consultant surgeons are employed within a general surgical subspecialty, including upper gastrointestinal, hepatobiliary, breast and endocrine, and colorectal. All surgeons from each team partake in the general surgery on-call roster. Each surgeon was included at least once within the observation periods. Experience of consultant surgeons ranged from 5 years of postfellowship experience to surgeons with more than 30 years of experience, with the majority having more than 10 years of postfellowship experience.

Patients were stratified into 2 distinct cohorts upon consultant review: smartphone positive (spP) was defined as a patient who was using a smartphone or who had their phone on their bed; a patient was classified as smartphone negative (spN) if they did not fulfil these criteria. The presence or absence of a smartphone was recorded by the authors, who were present on consultant ward rounds but not involved in the decision-making process of patient care. In order to minimize bias, only 1 surgeon (PGD) was aware that the study was being conducted and all patients were blinded to the study. Additional information that was collected included patient demographics, requirement for surgery, and length of stay (LOS). A patient who was discharged on the same day as the consultant review was considered to be discharged on day 1, all other patients were considered to have LOS greater than 1 day. Requirement for surgery was defined as a patient who underwent a surgical procedure in an operating suite. Thirty-day unplanned readmission rates for all patients were examined. Readmission to another public hospital within the state was also included within the readmission data.

\section{Observation Periods}

An initial 4-week pilot study was conducted to assess for a possible association between spP and same-day discharge. A second 8-week study period was undertaken 1 year later accounting for the employment of the authors at the study's institution. Unless stated, the results described are the accumulation of both study periods.

\section{Statistical Analysis}

As this is the first study of its kind, no prior estimates of numbers were known. After 2 weeks of data collection, data were analyzed in order to provide an estimate of the total number of patients required to provide a statistically valid result $(\alpha=0.05$; power $=0.80$ ). Sample size was calculated to be 40 subjects. It was agreed that in order to make the study as robust as possible, data should be collected for the 2 observation periods.

Demographic data are presented as means with standard deviations (SDs) or frequencies with percentages. A 2-sample Student $t$ test was used to compare the age of spP and $\mathrm{spN}$ patients. A $\chi^{2}$ test and logistic regressions were used to assess the association between smartphone status and patient demographics, LOS, and requirement for surgery. Results are presented as odds ratios (ORs) with 95\% confidence intervals (Cls). A $P$ value of $<.05$ was considered significant. All data were analyzed by using R 3.2.3 (R Foundation for Statistical Computing, Vienna, Austria).
TABLE 1. Demographic Data

\begin{tabular}{lcc}
\hline Characteristic & Period 1 & Period 2 \\
\hline Patients & 67 & 154 \\
\hline Male (\%) & $39(58.2 \%)$ & $90(58.4 \%)$ \\
\hline Mean age & 44.9 & 52.6 \\
\hline SpP (\%) & $13(19.4 \%)$ & $12(7.8 \%)$ \\
\hline Discharge day 1 & $21(31.35 \%)$ & $31(20.1 \%)$ \\
\hline Surgery & $3(4.5 \%)$ & $57(37.0 \%)$ \\
\hline NOTE: Abbreviation: spP, smartphone positive. & & \\
\hline
\end{tabular}

\section{RESULTS}

During the 2 observation periods, a total of 227 eligible surgical admissions were observed with complete data for 221 patients. Six patients were excluded as their smartphone status was not recorded. The study sample represents our population of interest within an ASU, and we had complete data for $97.4 \%$ of participants with a $100 \%$ follow-up. There was no significant effect of study between the 2 observation periods $\left(\chi^{2}=140.19 ; P=.10\right)$. The mean age of patients was 50.24 years. Further demographic data are presented in Table 1. Twenty-five (11.3\%) patients were spP and 196 (88.7\%) were spN. Fifty-two (23.5\%) patients were discharged home on day 1 , and 169 (76.5\%) had admissions longer than 1 day (see Figure). Sixty (27\%) patients underwent surgery during their admission. Twenty-two patients had unplanned readmissions; only 1 of these patients had been observed to be spP.

There was a statistically significant difference in ages between the spP and spN groups $(t=8.40 ; P<.0005)$, with the average age of spP patients being 31.84 years compared with 52.58 years for spN patients. There was no statistical difference between gender and smartphone status $\left(\chi^{2}=1.78 ; P=.18\right.$; Table 2).

For those patients discharged home on day 1, there was a statistically significant association with being $\operatorname{spP}\left(\chi^{2}=14.55\right.$, $P=.0001)$. Patients who were spP were 5.29 times more likely to be discharged on day $1(95 \% \mathrm{Cl}, 2.24-12.84)$. Of the variables analyzed, only gender failed to demonstrate an effect on discharge home on day 1 (Table 3). Overall, the presence of a smartphone was found to have a sensitivity of $56.0 \%(95 \% \mathrm{Cl}, 34.93-75.60)$ and a specificity of $80.6 \%(95 \%$ $\mathrm{Cl}, 74.37-85.90)$ in regard to same-day discharge. However, it was found to have a negative predictive value of $93.49 \%$ (95\% $\mathrm{Cl}$, 88.65-96.71).

When examining readmission rates, only $4 \%$ of spP patients were readmitted versus $10.7 \%$ of spN patients. Accounting for variables, spP patients were 4 times less likely to be readmitted, though this was not statistically significant (OR 4.02; $95 \% \mathrm{Cl}, 0.43-37.2 ; P=.22)$. Furthermore, when examining only those patients discharged on day 1, smartphone status was not a predictor of readmission (OR 0.94; $95 \% \mathrm{Cl}, 0.06-15.2$; $P=.97)$ 


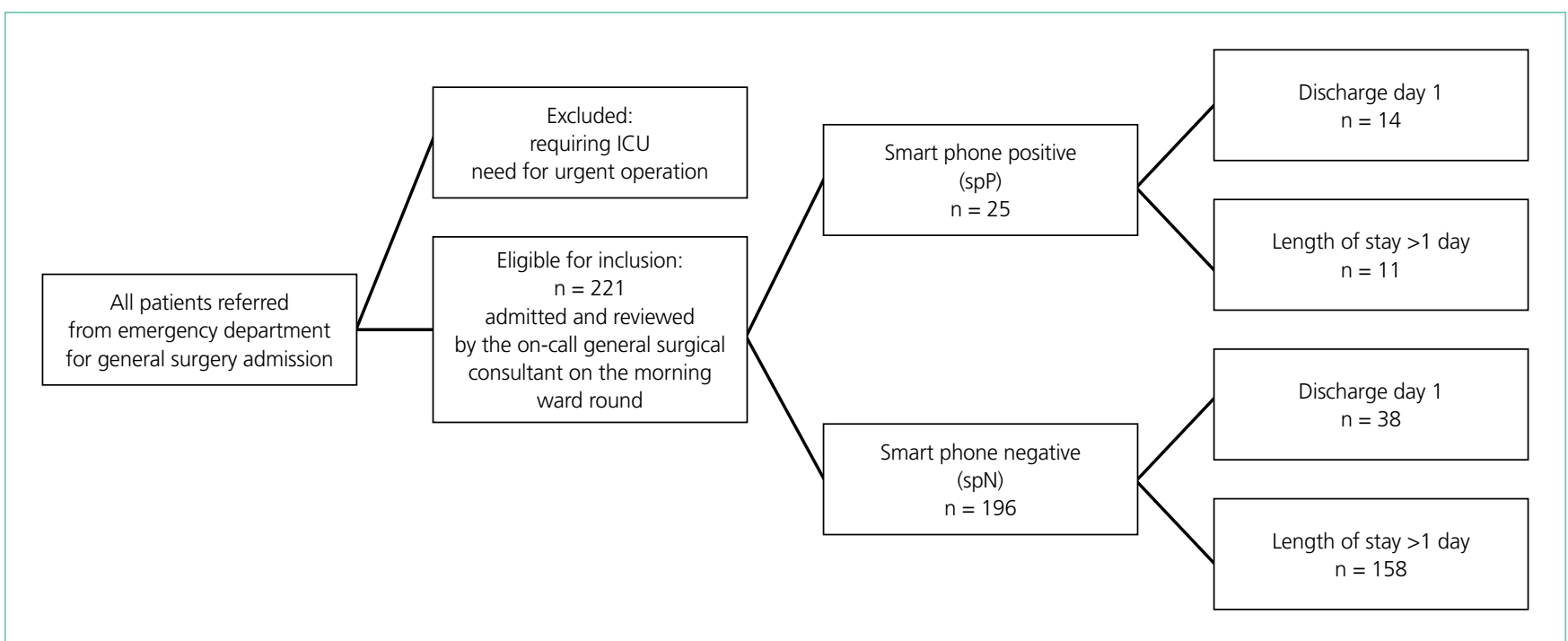

FIG. Patient pathway flow diagram.

NOTE: Abbreviations: ICU, intensive care unit; spP, smartphone positive; spN, smartphone negative.

TABLE 2. Smartphone Positive and Negative According to Gender and Age

\begin{tabular}{lccc}
\hline Characteristics & spP & spN & P value \\
\hline Male $(\%)$ & $11(44 \%)$ & $118(60.2 \%)$ & $\chi 2=1.78 ; P=.18$ \\
\hline Age & 31.84 & 52.58 & $t=8.40 ; P<.0005$ \\
\hline
\end{tabular}

NOTE: Abbreviations: spP, smartphone positive; spN, smartphone negative.

To mitigate the effect of age, analysis was conducted excluding those aged over 55 years (the previous retirement age in Australia), leaving 131 patients for analysis. The average age of spP patients was 31.8 years (SD 10.0) compared with 36.7 years (SD 10.9) for spN patients, representing a significant difference $(t=2.14 ; P=.04) ; 51.1 \%$ of patients were male, $19.1 \%$ of patients were spP, $26.0 \%$ of patients proceeded to an operation, the oldest spP was 51 years, and $29.0 \%$ of patients were discharged home on day 1 . There was no difference in gender and smartphone status $\left(\chi^{2}=0.33 ; P=.6\right)$. When analyzing those discharged on day 1 , again spP patients were more likely to be discharged home $\left(\chi^{2}=9.4 ; P=.002\right)$, and spP patients were 3.6 times more likely to be discharged home on day 1 .

There were $4 \mathrm{spP}$ patients who underwent an operation. Two patients had an incision and drainage of a perianal abscess, 1 patient underwent a laparotomy for an internal hernia after recently undergoing a Roux-en-Y gastric bypass at another hospital, and the final patient underwent a laparoscopic appendicectomy. One of these patients was still discharged home on day 1.

\section{DISCUSSION}

As J. A. Lindsay" said, "For one mistake made for not knowing, ten mistakes are made for not looking." At medical school, we are taught the finer techniques of the physical examination in order to support our diagnosis made from the history. It is not
TABLE 3. Statistical Analysis of those Discharged on Day 1

\begin{tabular}{lll}
\hline Characteristics & Odds Ratio & $\mathbf{9 5 \% \mathbf { C l }}$ \\
\hline Sex & 1.53 & $0.77-3.18$ \\
\hline Age & 0.98 & $0.96-1.00$ \\
\hline Operation & 1.64 & $0.046-0.46$ \\
\hline spP & 4.03 & $1.54-10.94$ \\
\hline
\end{tabular}

NOTE: Abbreviations: $\mathrm{Cl}$, confidence interval; spP, smartphone positive.

until we are experienced clinicians do we develop the clinical acumen and ability to tell an unwell patient from a well patient at a glance-colloquially known as the "end of the bed" assessment. In the pretechnology era, a well patient could frequently be seen reading their book, eg, the "novel-sign." With the advent of the smartphone and electronic devices upon which novels can be read, statuses updated, and locations "checked into" (ie, the modern "vital signs"), the book sign may be a thing of the past. However, the ability for the clinician to assess a patient's wellness is still crucial, and the value of any additional "physical signs" need to be estimated.

We observed a cohort of patients through a busy ASU in a tertiary hospital in South Australia, Australia. Acute surgical patients admitted to the hospital who were observed to be on their phones upon consultant review were more than 5 times likely to be discharged that same day. To the best of our knowledge, this is the first study to prospectively collect data to assess a frequently used but unevaluated clinical observation.

The use of a smartphone can tell us a lot about an individual's physiology. We can assume the individual's airway and breathing are adequate, allowing enough oxygen to reach the lungs and subsequently circulate. The individual is usually sit- 
ting up in bed and thus has an adequate blood pressure and blood oxygenation that can maintain cerebral perfusion. They have the cognitive and cerebral processing in place to function the device, and we can examine their cerebellar function by looking for fine-motor movements.

Mobile phone ownership is pervasive within Australia, ${ }^{5}$ with a conservative estimated $85.7 \%$ of the population $(20.57$ million people of a total population of approximately 24 million) owning a mobile phone and an estimated $50 \%$ to $79 \%$ of mobile phone ownership being of a smartphone. ${ }^{6,7}$ This ownership is not just limited to the young, with $74 \%$ of Australians over 65 owning or using a mobile phone. ${ }^{8}$ Despite this high phone ownership among those over 65, it is still significantly less than their younger counterparts and may be one reason for the absence of spP in those older than 51 years. A key point in the study is that overall phone ownership was not known, and, thus, it is not possible to determine the proportion of spN patients who were negative because they did not own a phone. However, based on general population data, the incidence of spP patients was well below that seen in the community $(11.3 \%)^{5}$ and even when excluding those over 55 , the percentage of spP patients only rose to $19.1 \%$. Unsurprisingly, increasing age was associated with a decreased likelihood of being spP $(P<.0005)$, as younger people are more likely to own a phone. ${ }^{8}$ There was no association with gender $(P=.18)$. There are a number of explanations that may explain the lower than expected percentage of spP patients, including the inability for the patient to gather their possessions during a medical emergency, patients storing their phones prior to doctor review (72\%-85\% of Australians report talking on phones in public places to be rude or intrusive ${ }^{5}$ ), but more importantly, that our hypothesis that patients were too unwell to use their device appears to hold true.

There are potential alternate reasons other than smartphone status that may account for patients being discharged home on day 1 . While there was no association seen with gender, the need for an operation prolonged a patient's stay (OR 1.64; 95\% $\mathrm{Cl}, 0.046-0.46)$, and there was a trend seen with increasing age (OR 0.98; 95\% Cl, 0.96-1.00). Neither of these 2 demographics are unsurprising: increasing age is associated with increasing medical comorbidities and thus complexity; even the simplest of operations require a postprocedure observation period, automatically increasing their LOS. Additionally, measured demographics are limited and there may be further unmeasured reasons that account for earlier discharge.

The other key component to this study is the value of the physical examination, albeit only assessing 1 component: the general inspection. In their review of the value of the physical examination of the cardiovascular system, Elder et al. highlight an important point: in traditional teaching, the value of a physical sign is compared with a diagnostic reference, typically imaging or an invasive test. ${ }^{9}$ They argue that this definition undervalues the physical examination and list other values aside from accuracy including accessibility, contribution to clinical care beyond diagnoses, cost effectiveness, patients' safety, patients' perceptions, and pedagogic value; and they argue that the physical examination should always be considered in regard to the clinical context-in this case, the newly admitted general surgical patient.

The assessment of the presence or absence of a smartphone is readily performed upon general inspection and is easily visible; general inspection of the patient and failure to observe the clinical sign when present are 2 of the greatest errors associated with physical examination. ${ }^{10}$ Furthermore, given its unique status as a physical sign, the authors' opinion and experience is that it is readily teachable. McGee states, "... a fundamental lesson [in regards to teaching] is that the diagnosis of many clinical problems, despite modern testing, still depends primarily on what the clinician sees, hears, and feels." ${ }^{11}$ In their article, Paley et al. found that more than $80 \%$ of patients admitted from the ED under internal medicine could be accurately diagnosed based largely on history and examination alone and concluded that basic clinical skills are sufficient for achieving an accurate diagnosis in most cases. ${ }^{12}$ Although Paley et al. were assisted with basic tests (such as electrocardiogram and basic haematological and biochemistry results), the point of clinical skills is not lost. Furthermore, this assessment was made in a group of patients generally considered to be complex in contrast to the "standard" appendicitis or cholecystitis patient that makes up a significant proportion of general surgical patients.

There are a number of limitations to this study, however, including smartphones that may have been missed during the observational period. Potential confounding variables such as socioeconomic status and the overall smartphone ownership of our subjects were not known. We did not ask all admitted patients whether they owned a phone or whether they had a phone in their possession. Knowledge of those who owned phones but were not in possession of them could strengthen our argument that spN patients were not using their phone because they were unwell, rather than just not having access to it.

However, this study has a number of strengths, including a large sample size and data that were prospectively collected by a method and in a setting that was the same for all participants. Clear and appropriate definitions were used, which minimizes misclassification bias. Participants and decision makers were blinded to the study, and potentially confounding variables such as age and sex were accounted for.

Assessing the suitability for discharge from the hospital is a decision encountered by hospital-based clinicians every day. These skills are not taught, but are rather learned as a junior doctor acquires experience. It is unlikely that protocols will be developed to aid identification of potential discharges from an acute surgical ward; acute surgical conditions are too varied and dynamic to be able to pool all data. We continue to rely on our own and fellow colleagues' (doctors, nurses, and other staff) input and assessment. However, our study has shown that it is possible to identify and quantify clinical findings that are already regularly used, albeit potentially subconsciously, to assess suitability for discharge. We have shown in this large, prospectively collected observational study that if a surgical patient is seen using their electronic device, they are more like- 
ly to be safe to go home. Thus, surgeons can reliably use this observation as a trigger to consider discharging the patient following a more thorough assessment.

\section{CONCLUSION}

While these observations might appear to be rather a simplistic way of trying to quantify whether or not a patient is fit for discharge, any clues that hint towards a patient's well-being should be taken into account when making an overall assessment. The active use of a smartphone is one such measure.

\section{References}

1. Sprivulis PC, Da Silva JA, Jacobs IG, Frazer AR, Jelinek GA. The association between hospital overcrowding and mortality among patients admitted via Western Australian emergency departments. Med J Aust. 2006;184(5): 208-212.

2. Shepherd T. Hospital Overcrowding kills as many as our road toll. The Advertiser. November 23, 2010. Available from: http://www.adelaidenow.com.au/ news/south-australia/hospital-overcrowding-kills-as-many-as-our-road-toll/ news-story/3389668c23b8b141f1d335b096ced416. Accessed February 2, 2017.

3. Shepperd S, Lannin NA, Clemson LM, McCluskey A, Cameron ID, Barras SL. Discharge planning from hospital to home. Cochrane Database Syst Rev. 2013;Jan 31(1):CD000313.

4. Breathnach CS, Moynihan JB. James Alexander Lindsay (1856-1931), and his clinical axioms and aphorisms. Ulster Med J. 2012;81(3):149-153.

5. Enhanced Media Metrics Australia. Product Insights Report. Digital Australia: A snapshot of attitudes and usage. August 2013. Ipsos Australia. North Sydney, Australia. Report available from: https://emma.com.au/wp-content/ uploads/2013/10/digital.pdf

6. Australian Communications and Media Authority. Communications report 2013-24. Melbounre: Commonwealth of Australia; 2014. http://www.acma. gov.au/ /media/Research\%20and\%20Analysis/Publication/Comms\%20

\section{Acknowledgments}

The authors thank Emma Knight and Nancy Briggs from the Data Management \& Analysis Centre, Discipline of Public Health, University of Adelaide.

Disclosure: No author nor the institution received any payment or services from a third party for any aspect of the submitted work and report no conflict of interest. There are no reported financial relationships with any entities by any of the authors. There are no patents pending based upon this publication. There are no relationships or activities that readers could perceive to have influenced, or give the appearance of influencing, the submitted work. The corresponding author is not in receipt of a research scholarship. The paper is not based on a previous communication.

Report\%202013\%2014/PDF/Communications\%20report\%20201314 LLOW-RES\%20FOR\%20WEB\%20pdf.pdf

7. Drumm J, Johnston S. Mobile Consumer Survery 2015-The Australian Cut. Deloitte. Australia; 2015. Deloitte Touche Tohmatsu. Sydney, Australia. file:///C:/Users/user/Desktop/deloitte-au-tmt-mobile-consumersurvey-2015-291015.pdf

8. Older Australians Resist Cutting the Cord: Australian Communications and Media Authority. 2014. http://www.acma.gov.au/theACMA/engage-blogs/ engage-blogs/Research-snapshots/Older-Australians-resist-cutting-the-cord. Accessed February 23, 2017.

9. Elder A, Japp A, Verghese A. How valuable is physical examination of the cardiovascular system? BMJ. 2016;354:i3309

10. Verghese A, Charlton B, Kassirer JP, Ramsey M, loannidis JP. Inadequacies of physical examination as a cause of medical errors and adverse events: a collection of vignettes. Am J Med. 2015;128(12):1322-1324.e3.

11. McGee S. A piece of my mind. Bedside teaching rounds reconsidered. JAMA. 2014;311(19):1971-1972.

12. Paley L, Zornitzki T, Cohen J, Friedman J, Kozak N, Schattner A. Utility of clinical examination in the diagnosis of emergency department patients admitted to the department of medicine of an academic hospital. Arch Intern Med. 2011;171(15):1394-1396. 